\title{
WHAT CAN WE LEARN FROM NEARBY AGNS?
}

\author{
LUIS C. HO \\ The Observatories of the Carnegie Institution of Washington \\ 813 Santa Barbara Street \\ Pasadena, CA 91101-1292, USA
}

\begin{abstract}
This contribution reviews the properties of nuclear activity in nearby galaxies, with emphasis on their implications for the demography of nuclear black holes and the nature of accretion flows in the regime of very low accretion rates.
\end{abstract}

\section{Why Study Nearby AGNs?}

In a meeting largely devoted to surveys of distant, luminous AGNs, it is instructive to examine what we know about AGNs in very nearby galaxies. Nearby AGNs are important for several reasons. Since AGNs derive their power from accretion onto central black holes, the statistics of AGNs serve as a crude surrogate to delineate the demography of massive black holes in galaxies - a topic of considerable recent interest (see Ho 2004a) - which is otherwise accessible only through painstaking kinematical observations. With the growing realization that black holes, and thus AGN activity, are part and parcel of the life-cycle of many galaxies, there is growing urgency to bridge the substantial gap that now exists between our knowledge of the space density of luminous quasars and that of quasar remnants. The extant information on the luminosity function of AGNs fainter than, say, $M_{B} \approx$ -20 to $-23 \mathrm{mag}$, is very sketchy at virtually any redshift, being practically nonexistent for $M_{B} \gtrsim-18$ mag. A census of nearby AGNs can place a robust constraint on the very faint end of the local $(z \approx 0)$ AGN luminosity function. By virtue of their proximity, nearby AGNs also offer a special vantage point to probe with high linear resolution the properties of their host galaxies and local environment. This level of detail is indispensable, for example, if one wishes to understand the triggering or fueling mechanisms for AGNs. Finally, as the evolutionary endpoint of quasars, nearby AGNs present an opportunity to study black hole accretion in a unique regime of parameter space, namely when the accretion drops to exceedingly low 


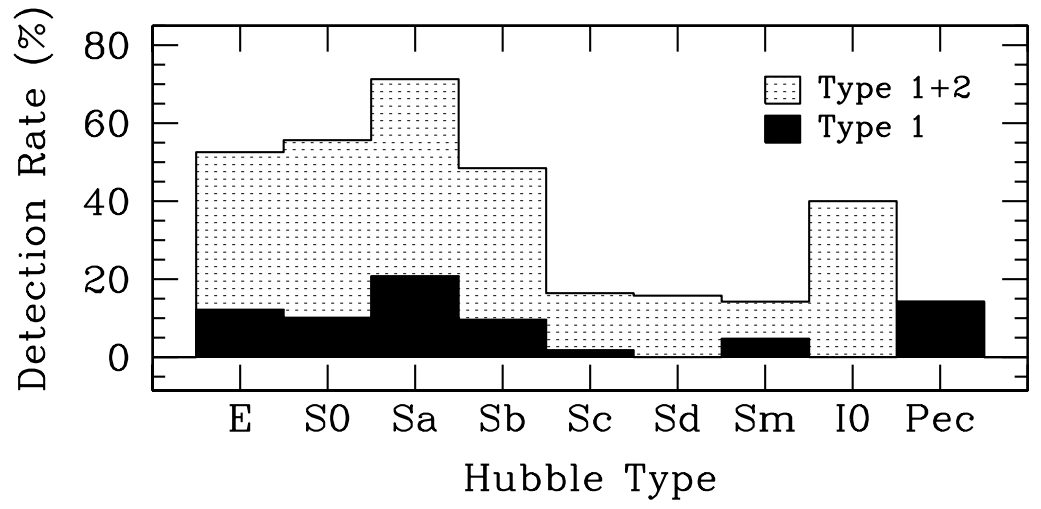

Figure 1. Detection rate of AGNs as a function of Hubble type in the Palomar survey. "Type 1" AGNs (those with broad $\mathrm{H} \alpha$ ) are shown separately from the total population (types 1 and 2). (Adapted from Ho et al. 1997.)

rates. Much emphasis has been placed on how AGNs turn on; it is just as important to understand how they turn off.

\section{Demography of Central Black Holes from AGN Surveys}

To the extent that an AGN signature signifies accretion onto a massive black hole, a local AGN census gives us a lower limit on the fraction of nearby galaxies hosting massive black holes (see Ho 2004b). There are many ways to select AGNs. Most surveys rely on selection criteria that isolate some previously known characteristics of AGNs. A common strategy employs color cuts to highlight the blue continuum typically present in AGN spectra. Some use objective prism plates to identify objects with strong and/or broad emission lines. Other wavelength-specific techniques to find candidate AGNs include preselection by radio or X-ray emission, or by "warm" infrared colors. Variability is also occasionally used. While all of these techniques have been successful, each introduces its own biases, and all ultimately require follow-up optical spectroscopy to confirm the AGN identification, to classify its type, and to determine its redshift.

Nearby AGNs are generally intrinsically faint. For these sources, most of the above-mentioned "tell-tale signs" are difficult or impossible to detect, either because they are severely diluted by the host galaxy or because they are intrinsically absent. To find local AGNs effectively, one has little recourse but to resort to brute force: direct spectroscopy of a magnitude- 
limited sample of galaxies. The following discussion draws mainly from the Palomar spectroscopic study of nearby galaxies (Ho, Filippenko, \& Sargent 1997, and references therein), which remains the most sensitive survey of its kind. The AGN samples emerging from the Sloan Digital Sky Survey (SDSS; Miller et al. 2003; Hao \& Strauss 2004; Kauffmann et al. 2004) certainly eclipse the Palomar sample in size, and they provide much better statistics on sources with moderate and high luminosities, but they do not go as deep on the faint end of the luminosity function. Moreover, as emphasized by Ho (2004b), the relatively large physical scale sampled by the SDSS spectra complicates the interpretation of LINERs, locally the dominant constituent of the AGN population.

Figure 1 gives an overview of the AGN detection rate in the Palomar survey. For conciseness, I do not distinguish the different subclasses of objects (Seyferts, LINERs, and transition objects), nor will I discuss the AGN "pedigree" of each; these topics have been recently reviewed elsewhere (Ho 2004b). The most pertinent points to draw from the figure are:

- AGNs are very common in nearby galaxies. At least $40 \%$ of all galaxies (with $B_{T} \leq 12.5 \mathrm{mag}$ ) emit AGN-like spectra.

- The detectability of AGNs depends strongly on the morphological type of the galaxy, being most common in early-type systems (E-Sbc). The detection rate of AGNs reaches $50 \%-75 \%$ in ellipticals, lenticulars, and bulge-dominated spirals but drops to $\lesssim 20 \%$ in galaxies classified as Sc or later.

- These AGN detection rates support the notion, popularized by dynamical studies, that central black holes are common in galaxies, and perhaps ubiquitous in those with bulges. This consistency check is important, and the apparent agreement should not be taken for granted, because direct dynamical detections of black holes are technically challenging and still limited by small number statistics.

\section{Evidence for Intermediate-mass Black Holes}

The distribution of black hole masses based on direct dynamical measurements (see, e.g., Tremaine et al. 2002) currently does not extend below $\sim 3 \times 10^{6} M_{\odot}$, the mass of the central black hole in the Milky Way. The record holder may be NGC $4945\left(\sim 1 \times 10^{6} M_{\odot}\right.$; Greenhill, Moran, \& Herrnstein 1997), but the kinematics of its nuclear $\mathrm{H}_{2} \mathrm{O}$ maser disk are not straightforward to interpret. How far does the bottom end of the mass 

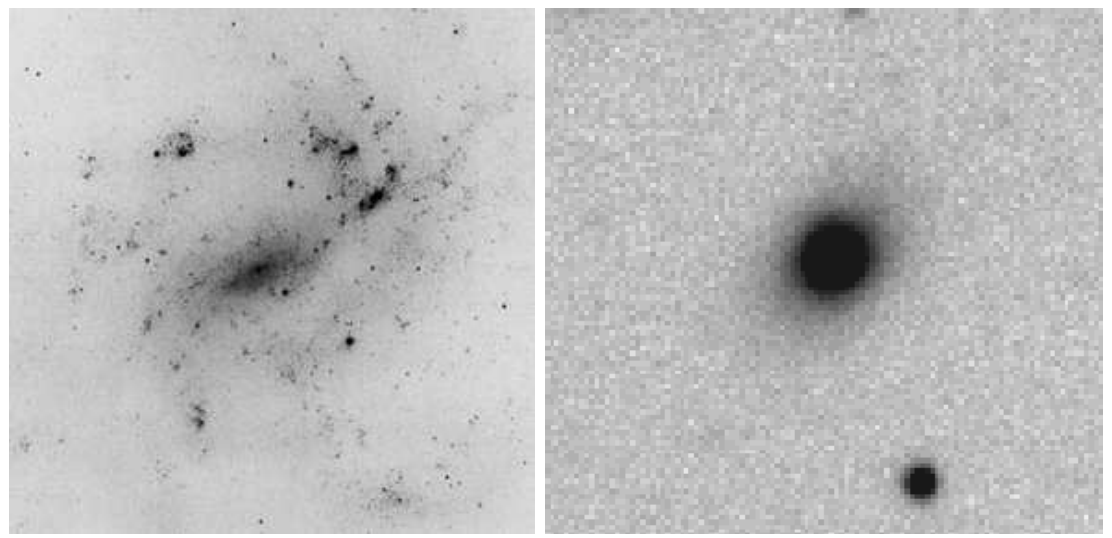

Figure 2. Two examples of AGNs in late-type galaxies. The left panel shows an optical image of NGC 4395, adapted from the Carnegie Atlas of Galaxies (Sandage \& Bedke 1994 ); the image is $\sim 15^{\prime}$ (17 kpc) on a side. The right panel shows an $R$-band image of POX 52, adapted from Barth et al. (2004); the image is $\sim 25^{\prime \prime}(11 \mathrm{kpc})$ on a side.

function of black holes extend? Is there a sharp boundary between the masses of stellar and nuclear black holes? The current limit of $\sim 10^{6} M_{\odot}$ most likely reflects our present observational sensitivity rather than a physical threshold. Intermediate-mass $\left(\sim 10^{3}-10^{5} M_{\odot}\right)$ black holes, if they exist, may offer important clues to the nature of the "seeds" of supermassive $\left(10^{6}-10^{9} M_{\odot}\right)$ black holes. The inspiral of smaller black holes onto bigger ones, an inevitable consequence of hierarchical structure formation, may also provide a significant contribution to the integrated gravitational radiation background (e.g., Hughes 2002).

To date, the only known cases of intermediate-mass black holes based on resolved kinematics come from studies of globular clusters (Gebhardt, Rich, \& Ho 2002; Gerssen et al. 2002), which, in any case, have been controversial (Baumgardt et al. 2003a, b). Clearly, it would be desirable to establish whether intermediate-mass black holes exist in galactic nuclei. Nuclear intermediate-mass black holes would manifest themselves as AGNs of moderately low luminosities $\left(\lesssim 10^{43} \mathrm{erg} \mathrm{s}^{-1}\right)$, most likely in low-mass or very late-type galaxies.

Two such cases have recently been reported (Fig. 2). The nearby $(\sim 4$ Mpc), Magellanic spiral (Sdm) galaxy NGC 4395 has long been known to host a low-luminosity Seyfert 1 nucleus (Filippenko \& Sargent 1989), which has been well studied from radio to X-ray wavelengths. Filippenko \& Ho (2003) used several lines of evidence to argue that the mass of the central 


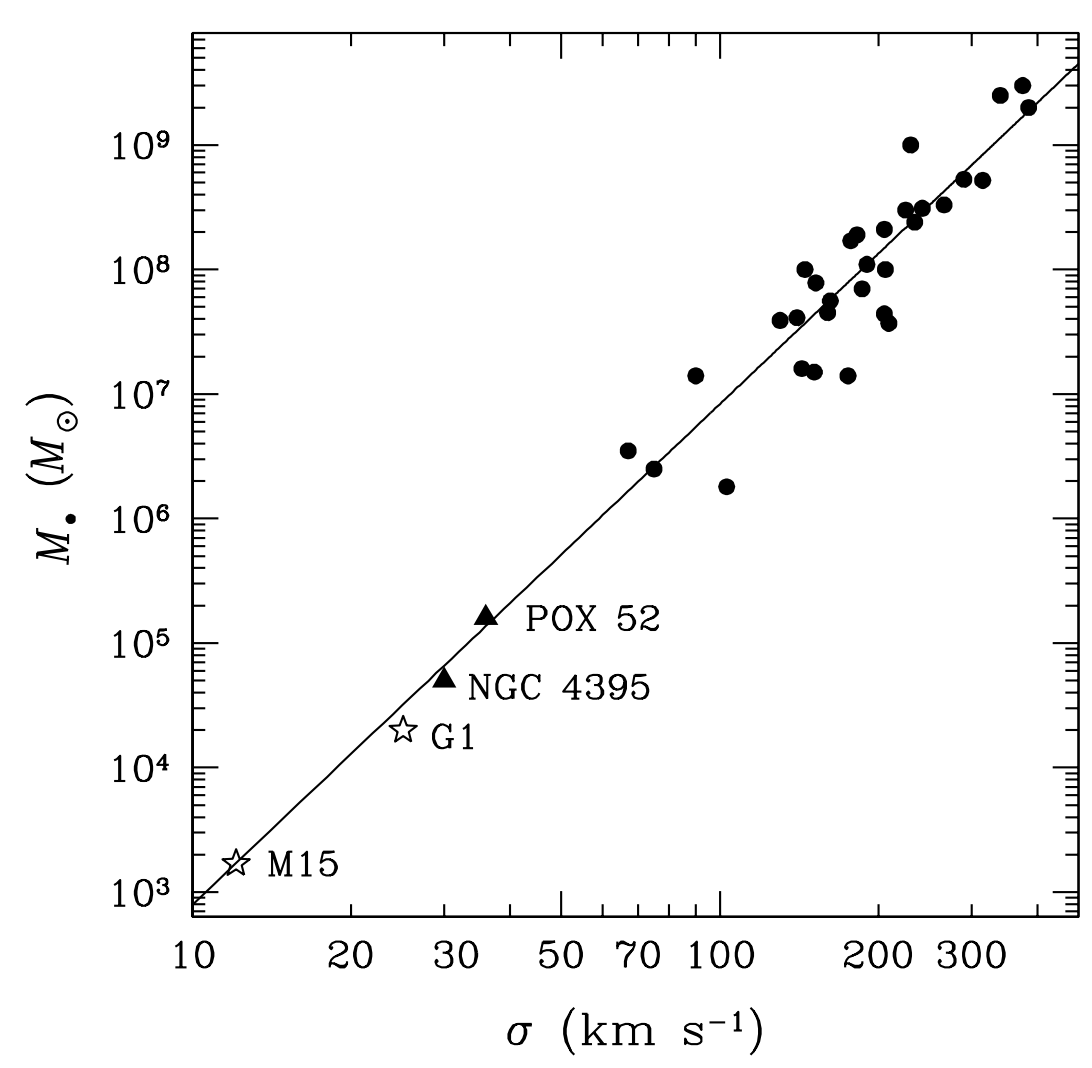

Figure 3. The black hole mass vs. velocity dispersion relation extended to the regime of intermediate-mass black holes. The dashed line shows the fit of Tremaine et al. (2002): $M_{\bullet} \propto \sigma^{4.02}$.

black hole in NGC 4395 lies in the range of $10^{4}-10^{5} M_{\odot}$. This result is significant because it demonstrates unequivocally that nuclear black holes can exist in a bulgeless galaxy.

Equally striking is POX 52, a considerably more distant $(\sim 90 \mathrm{Mpc})$ dwarf galaxy. Barth et al. (2004) find that POX 52 has an optical spectrum that is virtually indistinguishable from that of NGC 4395. It also shows tentative evidence of X-ray emission. Based on the velocity width of the broad Balmer lines and the continuum luminosity, Barth et al. obtain a virial mass of $\sim 10^{5} M_{\odot}$. Interestingly, POX 52 appears to a dwarf elliptical galaxy, the first known to host an unambiguous AGN. This is quite unexpected because dwarf elliptical galaxies, while technically spheroidal 
systems, bear little physical resemblance to classical bulges. Dwarf ellipticals occupy a distinct locus on the fundamental plane of hot stellar systems (Bender, Burstein, \& Faber 1992; Geha, Guhathakurta, \& van der Marel 2003), and they are thought to originate from harassment and tidal stripping of late-type (bulgeless) disk galaxies (e.g., Moore et al. 1996). Thus, like NGC 4395, POX 52 stands as testimony that a bulge is not a prerequisite for the formation of central black hole.

Finally, another surprise (Fig. 3). Although no firm conclusions can yet be drawn based on such meager statistics, it is intriguing, indeed mildly perplexing, that the four new candidate intermediate-mass black holes (the globular clusters M15 and G1 and the AGNs in NGC 4395 and POX 52) evidently seem to obey the relation between black hole mass and stellar velocity dispersion established by supermassive black holes (Gebhardt et al. 2000; Ferrarese \& Merritt 2000; Tremaine et al. 2002).

Are AGNs in dwarf or late-type galaxies common? Evidently not, at least in the nearby Universe. NGC 4395 is one of a kind in the Palomar survey of $\sim 500$ galaxies. On the other hand, POX 52 was discovered in an objective prism survey of a relatively small area of sky (Kunth, Sargent, \& Bothun 1987), so objects like it cannot be that rare. Using the first data release from the SDSS, Greene \& Ho (2004) have identified 150 AGNs that have sub- $10^{6} M_{\odot}$ black holes. The SDSS images do not furnish reliable information on the host galaxy morphologies, but a sizable fraction of them appear to be relatively disk-dominated spirals.

\section{The Optical Luminosity Function of $z \approx 0$ AGNs}

Many astrophysical applications of AGN demographics benefit from knowing the AGN luminosity function, $\Phi(L, z)$. Whereas $\Phi(L, z)$ has been reasonably well charted for high $L$ and high $z$ using quasars, it is very poorly known at low $L$ and low $z$. Indeed, until very recently there has been no reliable determination of $\Phi(L, 0)$. Since nearby AGNs are faint, disentangling the nuclear emission from the much brighter contribution of the host galaxy poses a major challenge. It is unacceptable to simply use the integrated emission from the entire galaxy. Moreover, most optical luminosity functions of bright, more distant AGNs are specified in terms of the nonstellar optical continuum (usually in the $B$ band), whereas spectroscopic surveys of nearby galaxies generally only reliably measure optical line emission (e.g., $\mathrm{H} \alpha$ ) because the featureless nuclear continuum is often impossible to detect in ground-based, seeing-limited apertures. 


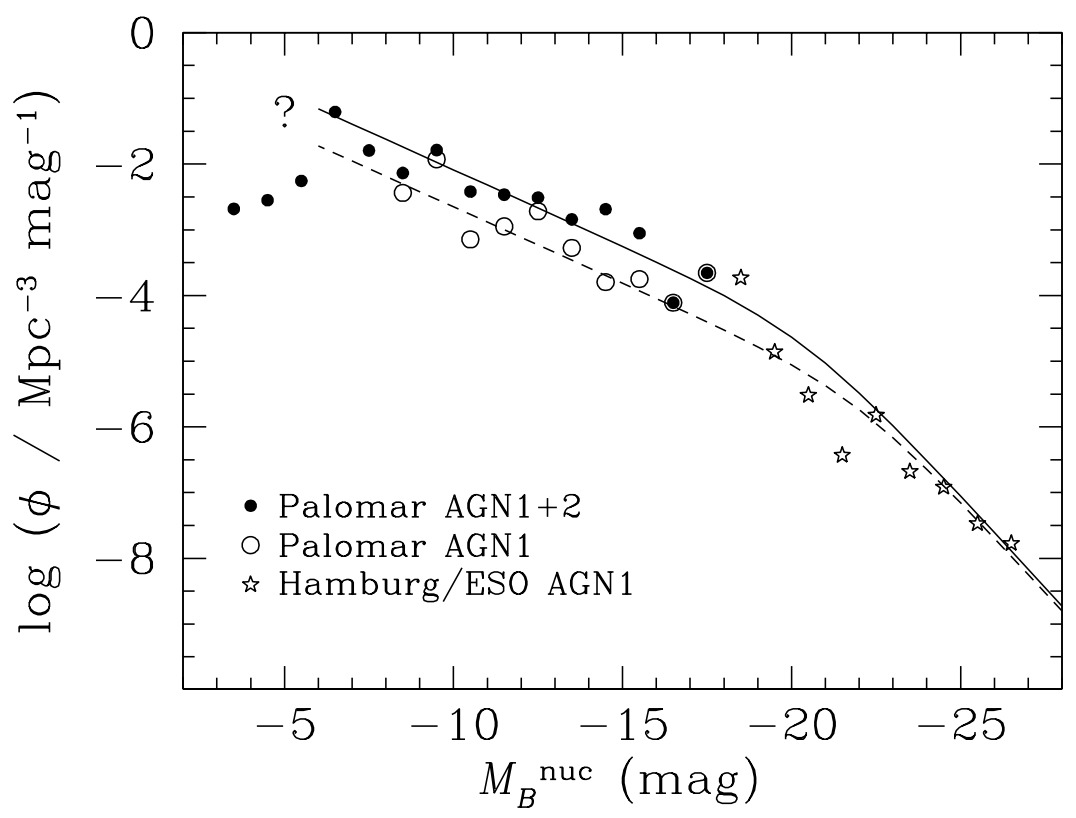

Figure 4. The B-band nuclear luminosity function of nearby AGNs derived from the Palomar survey. The filled circles include all (type $1+$ type 2 ) sources, while the open circles include only type 1 sources. The sample of luminous Seyfert $1 \mathrm{~s}$ and quasars from the Hamburg/ESO survey of Köhler et al. (1997) is shown as stars. A double power-law fit to the Palomar and Hamburg/ESO samples is shown as a solid (type $1+$ type 2) and dashed (type 1) curve. (Adapted from Ho 2004b.)

The strategy adopted for the Palomar survey utilizes the well-known correlation between Balmer emission-line luminosity and optical featureless continuum luminosity, which has been shown by Ho \& Peng (2001) to hold for low-luminosity AGNs. Figure 4 shows the $B$-band nuclear luminosity function for the Palomar AGNs. Two versions are presented, each representing an extreme view of what kind of sources should be regarded as bona fide AGNs. The open circles include only type 1 nuclei, sources in which broad $\mathrm{H} \alpha$ emission was detected and hence whose AGN status is incontrovertible. This may be regarded as the most conservative assumption and a lower bound, since genuine narrow-lined AGNs do exist. The solid circles lump together all sources classified as LINERs, transition objects, or Seyferts, both type 1 and type 2. This represents the most optimistic view and an upper bound, since undoubtedly some narrow-lined sources must be 
stellar in origin but masquerading as AGNs. The true space density of local AGNs most likely lies between these two possibilities. In either case, the differential luminosity function is reasonably well approximated by a single power law from $M_{B} \approx-5$ to $-18 \mathrm{mag}$, roughly of the form $\Phi \propto L^{-1.2 \pm 0.2}$. The slope may flatten for $M_{B} \gtrsim-7 \mathrm{mag}$, but the luminosity function is highly uncertain at the faint end because of density fluctuations in our local volume.

For comparison, I have overlaid the luminosity function of $z \lesssim 0.3$ quasars and Seyfert 1 nuclei as determined by Köhler et al. (1997) from the Hamburg/ESO UV-excess survey, scaled to the adopted cosmological parameters of $H_{0}=75 \mathrm{~km} \mathrm{~s}^{-1} \mathrm{Mpc}^{-1}, \Omega_{\mathrm{m}}=0.3$, and $\Omega_{\Lambda}=0.7$. This sample extends the luminosity function from $M_{B} \approx-18$ to -26 mag. Although the two samples do not strictly overlap in luminosity, it is apparent the two samples roughly merge, and that the break in the combined luminosity function most likely falls near $M_{B}^{*} \approx-19 \mathrm{mag}$, where the space density $\phi \approx 1 \times 10^{-4} \mathrm{Mpc}^{-3} \mathrm{mag}^{-1}$.

\section{Radiative Inefficient Accretion}

As Figure 4 shows, clearly the luminosities of most nearby nuclei are extremely low. To cast this statement in more physical terms, I have converted the optical luminosities to bolometric luminosities and then compared them relative to the Eddington luminosities, which were estimated from the black hole mass vs. velocity dispersion relation (Fig. 5). Note that nearly all the objects have $L_{\mathrm{bol}}<10^{44} \mathrm{erg} \mathrm{s}^{-1}$, and most significantly less. Seyferts are on average 10 times more luminous than LINERs or transition objects. More importantly, nearby nuclei are highly sub-Eddington systems. All objects have $L_{\mathrm{bol}} / L_{\mathrm{Edd}}<1$, with most $<10^{-3}$. One might argue that this is to be expected. After all, in the present-day Universe bulge-dominated galaxies have spent most of their gas supply, leaving little fuel for central accretion. But this is not the whole story.

It is true that not much fuel is needed to sustain the low level of activity observed. For a canonical radiative efficiency of $\eta=0.1$ appropriate for a geometrically thin disk, $L_{\mathrm{bol}}=10^{40}-10^{42} \mathrm{erg} \mathrm{s}^{-1}$ requires only $\dot{M}=10^{-6}-10^{-4} M_{\odot} \mathrm{yr}^{-1}$, ostensibly a minuscule amount. The trouble is that the innermost regions of early-type galaxies should have much larger gas reservoirs than this. Ho (2004, in preparation) estimates that most galactic nuclei should have gas supplies as large as $\dot{M} \approx 10^{-3} M_{\odot}$

$\mathrm{yr}^{-1}$, predominantly coming from mass loss from evolved stars and Bondi 

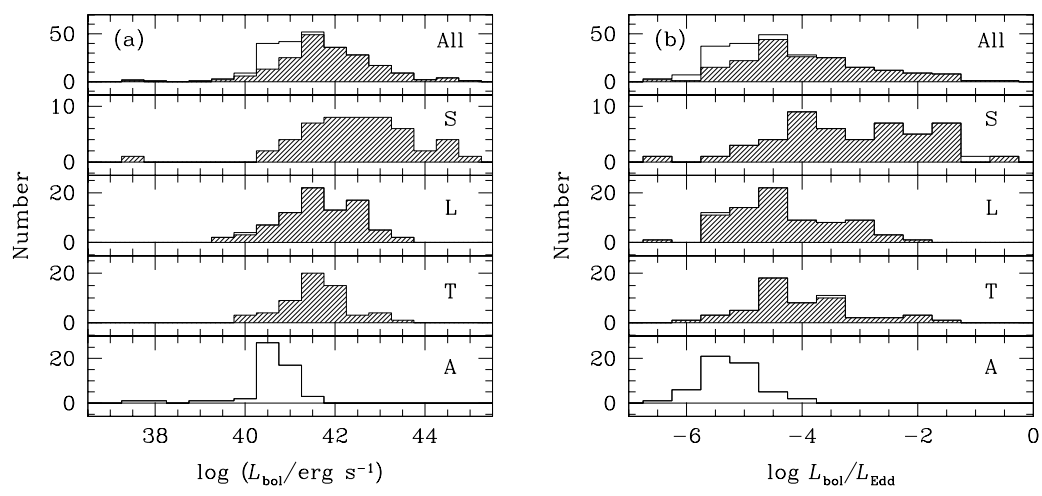

Figure 5. Distribution of (a) nuclear bolometric luminosities and (b) Eddington ratios $\lambda \equiv L_{\mathrm{bol}} / L_{\mathrm{Edd}} . \mathrm{S}=$ Seyferts, $\mathrm{L}=$ LINERs, $\mathrm{T}=$ transition objects, and $\mathrm{A}=$ absorptionline nuclei. Open histograms denote upper limits. (From Ho 2004b.)

accretion of hot gas. If this material were to be accreted and converted to radiation with $\eta=0.1$, nearby AGNs should be much more spectacular that observed. This suggests that either only a tiny fraction of the available gas gets accreted or that $\eta$ is much less than 0.1, as postulated in models of radiatively inefficient accretion flows (see Quataert 2001 for a review). If the gas is prevented from accreting, it is unlikely that supernova winds are the culprit, since there is little evidence of recent star formation in nearby nuclei (Ho et al. 2003). Instead, recent models of radiatively inefficient accretion flows suggest that these systems are naturally prone to generating winds or outflows, which would curtail the amount of material that actually gets accreted. Note, however, that this does not obviate the need for $\eta$ to be small - only that it does not have to be as small as it would be in the absence of winds - because a radiatively inefficient flow is a precondition for establishing the winds.

Nearby, low-luminosity AGNs are not simply scaled-down versions of luminous AGNs. As a direct consequence of their low accretion rates, most nearby AGNs do not have "standard" optically thick, geometrically thin accretion disks. Instead, their central engines are more akin to the class of radiatively inefficient accretion flows discussed in the recent literature. Additional arguments in support of this picture can be found in Ho (2003). 


\section{References}

1. Barth, A. J., Ho, L. C., Rutledge, R. E., \& Sargent, W. L. W. 2004, ApJ, in press

2. Baumgardt, H., Hut, P., Makino, J., McMillan, S., \& Portegies Zwart, S. 2003a, ApJ, 582, L21

3. Baumgardt, H., Makino, J., Hut, P., McMillan, S., \& Portegies Zwart, S. 2003b, ApJ, 589, L25

4. Bender, R., Burstein, D., \& Faber, S. M. 1992, ApJ, 399, 462

5. Ferrarese, L., \& Merritt, D. 2000, ApJ, 539, L9

6. Filippenko, A. V., \& Ho, L. C. 2003, ApJ, 588, L13

7. Filippenko, A. V., \& Sargent, W. L. W. 1989, ApJ, 342, L11

8. Gebhardt, K., et al. 2000, ApJ, 539, L13

9. Gebhardt, K., Rich, R. M., \& Ho, L. C. 2002, ApJ, 578, L41

10. Geha, M., Guhathakurta, P., \& van der Marel, R. P. 2003, AJ, 126, 1794

11. Gerssen, J., van der Marel, R. P., Gebhardt, K., Guhathakurta, P., Peterson, R. C., \& Pryor, C. 2002, AJ, 124, 3270 (Addendum: 2003, 125, 376)

12. Greene, J. E., \& Ho, L. C. 2004, ApJ, submitted

13. Greenhill, L. J., Moran, J. M., \& Herrnstein, J. R. 1997, ApJ, 481, L23

14. Hao, L., \& Strauss, M. A. 2004, in Carnegie Observatories Astrophysics Series, Vol. 1: Coevolution of Black Holes and Galaxies, ed. L. C. Ho (Pasadena: Carnegie Observatories)

15. Ho, L. C. 2003, in Active Galactic Nuclei: from Central Engine to Host Galaxy, ed. S. Collin, F. Combes, \& I. Shlosman (San Francisco: ASP), 379

16. Ho, L. C. 2004a, ed., Coevolution of Black Holes and Galaxies (Cambridge: Cambridge Univ. Press)

17. Ho, L. C. 2004b, in Carnegie Observatories Astrophysics Series, Vol. 1: Coevolution of Black Holes and Galaxies, ed. L. C. Ho (Cambridge: Cambridge Univ. Press), in press

18. Ho, L. C., Filippenko, A. V., \& Sargent, W. L. W. 1997, ApJ, 487, 568

19. Ho, L. C., Filippenko, A. V., \& Sargent, W. L. W. 2003, ApJ, 583, 159

20. Ho, L. C., \& Peng, C. Y. 2001, ApJ, 555, 650

21. Hughes, S. A. 2002, MNRAS, 331, 805

22. Kauffmann, G., et al. 2004, MNRAS, in press

23. Köhler. T., Groote, D., Reimers, D., \& Wisotzki, L. 1997, A\&A, 325, 502

24. Kunth, D., Sargent, W. L. W., \& Bothun, G. D. 1987, AJ, 92, 29

25. Miller, C. J., Nichol, R. C., Gomez, P. L., Hopkins, A. M., \& Bernardi, M. 2003, ApJ, 597, 142

26. Moore, B., Katz, N., Lake, G., Dressler, A., \& Oemler, A. 1996, Nature, 379, 613

27. Quataert, E. 2001, in Probing the Physics of Active Galactic Nuclei by Multiwavelength Monitoring, ed. B. M. Peterson, R. S. Polidan, \& R. W. Pogge (San Francisco: ASP), 71

28. Sandage, A., \& Bedke, J. 1994, The Carnegie Atlas of Galaxies (Washington, DC: Carnegie Inst. of Washington)

29. Tremaine, S., et al. 2002, ApJ, 574, 740 